\title{
The Role of Surgery in the Treatment of Bismuth-Corlette Type IV Perihilar Cholangiocarcinoma
}

\author{
Laura Alaimo, MD, Fabio Bagante, MD, PhD 말, and Andrea Ruzzenente, MD, PhD \\ Department of Surgery, Unit of Hepato-Pancreato-Biliary Surgery, University of Verona Medical School, Verona, Italy
}

\section{PAST}

Bismuth-Corlette (BC) type IV perihilar cholangiocarcinoma (pCCA) involving the second-order biliary ducts has traditionally been considered a locally advanced and unresectable tumor. In recent decades, several authors have proposed an aggressive surgical approach for the treatment of BC type IV pCCA, including extended hepatectomies (i.e., trisectionectomy) and vascular resections. ${ }^{1,2}$ Although these approaches might be associated with a higher risk of complications and in-hospital mortality, Eastern and Western authors recently reported that surgical resections with curative intent might represent a valid treatment option for these patients. ${ }^{1,3,4}$

\section{PRESENT}

We investigated the short- and long-term outcomes of 1138 patients who underwent surgical resection for BC type IV and type II-III pCCA at one of 20 Western hepatobiliary centers participating in the study. ${ }^{5}$ As could be expected, BC IV pCCA patients more frequently required preoperative portal vein embolization, extended hepatectomy, and vascular resections and also had increased incidence of positive margin (R1) compared with BC type II-III pCCA patients. Postoperatively, pCCA patients had similar incidence of severe (Clavien-Dindo $\geq 3$ ) complications (BC IV, $51.3 \%$ versus BC II-III, $45.8 \%, p=0.1$ ) and 90-day mortality (BC IV, $12.2 \%$ versus BC II-III, $13.4 \%, p=0.6)$. Interestingly, BC type was

(C) Society of Surgical Oncology 2021

First Received: 26 March 2021

Accepted: 26 March 2021;

Published Online: 21 April 2021

F. Bagante, $\mathrm{MD}, \mathrm{PhD}$

e-mail: fabio.bagante@gmail.com not associated with prognosis and, among N0/R0 patients, BC IV patients had 5-year overall survival of $41 \%$ compared with $43 \%$ for BC II-III patients ( $p=0.6)$.

\section{FUTURE}

Data from the current study demonstrate that, also in Western centers, surgery is a valid treatment option for BC type IV pCCA patients, providing similar short- and long-term outcomes when compared with surgery for BC type II-III patients. Innovative surgical approaches (i.e., minimally invasive surgery) and perioperative patient management (i.e., personalized targeted therapies) would further improve the surgical and oncological outcomes of BC type IV pCCA patients.

DISCLOSURES The authors declare no conflicts of interest.

\section{REFERENCES}

1. Ku D, Tang R, Pang T, Pleass H, Richardson A, Yuen L, et al. Survival outcomes of hepatic resections in Bismuth-Corlette type IV cholangiocarcinoma. ANZ J Surg. 2020;90(9):1604-14.

2. Mizuno T, Ebata T, Nagino M. Advanced hilar cholangiocarcinoma: an aggressive surgical approach for the treatment of advanced hilar cholangiocarcinoma-perioperative management, extended procedures, and multidisciplinary approaches. Surg Oncol. 2020;33:201-6.

3. Ebata T, Mizuno T, Yokoyama Y, Igami T, Sugawara G, Nagino M. Surgical resection for Bismuth type IV perihilar cholangiocarcinoma. Br J Surg. 2018;105(7):829-38.

4. Wiggers JK, Groot Koerkamp B, Cieslak KP, Doussot A, van Klaveren D, Allen PJ, et al. Postoperative mortality after liver resection for perihilar cholangiocarcinoma: development of a risk score and importance of biliary drainage of the future liver remnant. J Am Coll Surg. 2016;223(2):321-31e1.

5. Ruzzenente A, Bagante F, Olthof P B, Aldrighetti L, Alikhanov R, Cescon M, et al. Surgery for Bismuth-Corlette type IV perihilar cholangiocarcinoma: results from a western multicenter collaborative group. Ann Surg Oncol. 2021. https://doi.org/10.1245/s10434-021-09905-z.

Publisher's Note Springer Nature remains neutral with regard to jurisdictional claims in published maps and institutional affiliations. 